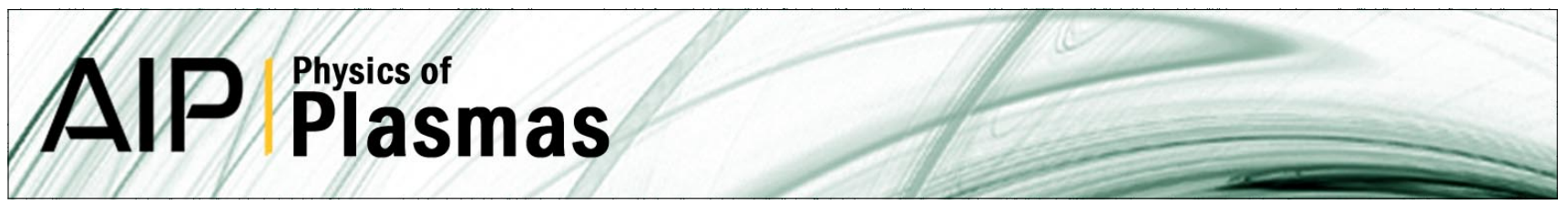

\title{
Towards a complete parametrization of the ordinary-mode electromagnetic instability in counterstreaming plasmas. I. Minimizing ion dynamics
}

D. Ibscher, M. Lazar, M. J. Michno, and R. Schlickeiser

Citation: Phys. Plasmas 20, 012103 (2013); doi: 10.1063/1.4774066

View online: http://dx.doi.org/10.1063/1.4774066

View Table of Contents: http://pop.aip.org/resource/1/PHPAEN/v20/i1

Published by the American Institute of Physics.

\section{Related Articles}

Pressure driven tearing and interchange modes in the reversed field pinch

Phys. Plasmas 20, 012119 (2013)

Roles of poloidal rotation in the $\mathrm{q}=1$ high-order harmonic tearing modes in a tokamak plasma

Phys. Plasmas 20, 012512 (2013)

Spatiotemporal structure of a millimetric annular dielectric barrier discharge plasma actuator Phys. Fluids 25, 017103 (2013)

Modulational instability of a Langmuir wave in plasmas with energetic tails of superthermal electrons Phys. Plasmas 20, 012115 (2013)

Time-domain simulation of nonlinear radiofrequency phenomena

Phys. Plasmas 20, 012116 (2013)

\section{Additional information on Phys. Plasmas}

Journal Homepage: http://pop.aip.org/

Journal Information: http://pop.aip.org/about/about_the_journal

Top downloads: http://pop.aip.org/features/most_downloaded

Information for Authors: http://pop.aip.org/authors

\section{ADVERTISEMENT}

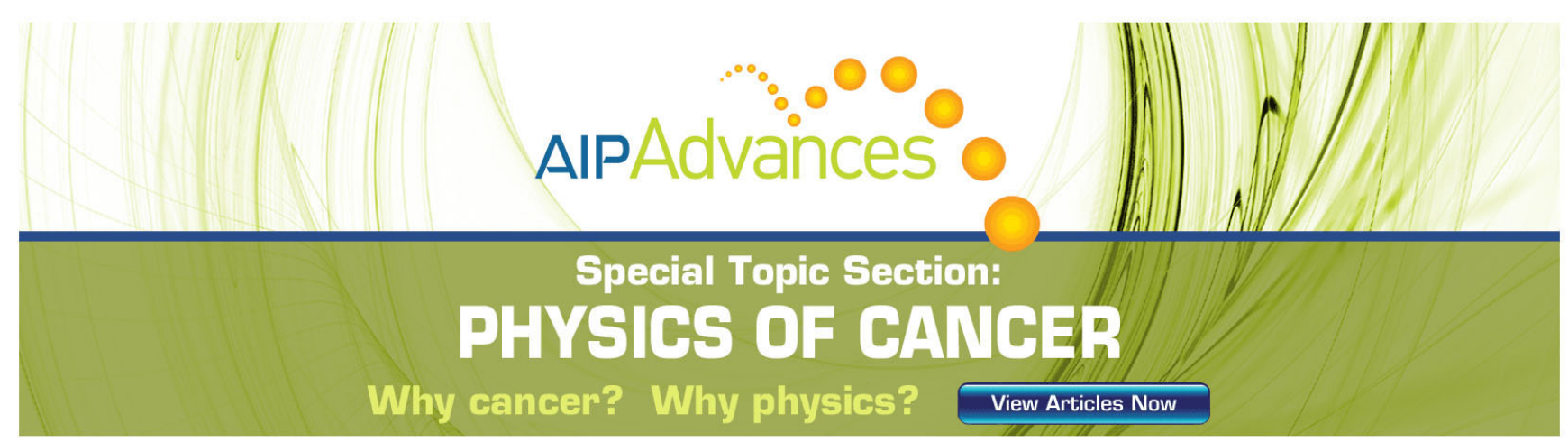




\title{
Towards a complete parametrization of the ordinary-mode electromagnetic instability in counterstreaming plasmas. I. Minimizing ion dynamics
}

\author{
D. Ibscher, ${ }^{1, a)}$ M. Lazar, ${ }^{1,2}$ M. J. Michno, ${ }^{1}$ and R. Schlickeiser ${ }^{1,3}$ \\ ${ }^{1}$ Institut für Theoretische Physik, Lehrstuhl IV: Weltraum-und Astrophysik, Ruhr-Universität Bochum, \\ D-44780 Bochum, Germany \\ ${ }^{2}$ Centre for Mathematical Plasma Astrophysics, Celestijnenlaan 200B, 3001 Leuven, Belgium \\ ${ }^{3}$ Research Department Plasmas with Complex Interactions, Ruhr-Universität Bochum, D-44780 Bochum, \\ Germany
}

(Received 29 October 2012; accepted 18 December 2012; published online 7 January 2013)

\begin{abstract}
The ordinary mode instability can be driven by drifting bi-Maxwellian plasma particle distributions with and without temperature anisotropy. Here, the linear instability analysis is generalized for realistic settings, when the plasma streams are magnetized and hot enough. The new parametrization proposed in this study enables a better understanding of the interplay of counterstreaming and temperature anisotropy, providing the derivation of new regimes of the ordinary mode instability. Accurate analytical forms are derived for the instability conditions for general values of the temperature anisotropy, the streaming velocity, and the parallel plasma beta. To keep the analysis straightforward, the role of ions is minimized. (C) 2013 American Institute of Physics. [http://dx.doi.org/10.1063/1.4774066]
\end{abstract}

\section{INTRODUCTION}

Countermoving plasma streams often occur in astrophysical sources, when supersonic plasma flows encounter obstacles, such as, e.g., the interaction of the solar wind with planetary magnetospheres and/or comets. The counterstreaming plasmas, as a special class of velocity anisotropic particle distribution function (VAP), are subject to a variety of kinetic plasma instabilities, and recent studies have shown an intriguing competition of the electromagnetic and electrostatic instabilities. ${ }^{1-7}$ The instabilities of VAPs, either in the form of countermoving beams or bi-Maxwellians with large enough temperature anisotropy, quickly dissipate their free energy in the form of electromagnetic fluctuations which are crucial for the formation of shock structures. At the same time, the initial unstable particle distribution function relaxes to intermediate quasi-stable distribution functions, which then might be subject to further secular instabilities.

Of particular interest in linear instability studies are the quickest kinetic plasma instabilities. In unmagnetized plasmas with counterstreaming beams, the prime competitors are the obliquely (with respect to the counterstream direction) propagating electrostatic waves, and the electromagnetic filamentation instability, which generates quasistationary astrophysical and laboratory seed magnetic fields. ${ }^{1,6}$ In plasmas with largescale ordered magnetic fields, the linear instability analysis becomes more involved. Most analytical studies are restricted to fluctuations with wave vectors parallel to the uniform magnetic field direction, ${ }^{8,9}$ or adopting idealized VAPs such as cold counterstreaming plasmas, ${ }^{10}$ symmetric counterstreams, ${ }^{11-14}$ or nonstreaming bi-Maxwellians and bi-kappa distributions.

Here, we investigate hot counterstreaming plasmas (of finite temperatures), which are not necessarily symmetric, but with arbitrary densities, parallel temperatures, temperature ani-

\footnotetext{
a)ibscher@tp4.rub.de.
}

sotropies, and streaming velocities. The new parametrization introduced in Sec. II satisfies the charge and current neutral conditions ${ }^{15} \Sigma_{s} \rho_{s}=\Sigma_{s} \mathbf{J}_{s}=0$, where $s$ states for the number of streams. Adopting the minimal restriction of equal perpendicular temperatures for the counterstreams, which reflects an axial symmetry of the total plasma system, the linear dispersion relation of the ordinary mode with perpendicular wave vector orientations decouples from the dispersion relation of the other competing modes. In Secs. III and IV, we investigate the ordinary mode instability for arbitrary plasma-beta values $\beta_{\|} \equiv 8 \pi n k_{B} T_{\|} / B_{0}^{2}$, to derive very accurate analytical formulas for the threshold conditions of marginal instability in a multispecies plasma. To minimize the role of heavier species (ions or protons) and keep the analysis tractable, we assume equal parameters characterizing the free energy of electrons and protons, i.e., $T_{e} \simeq T_{p}$ and $m_{e} V_{e}^{2} \simeq m_{p} V_{p}^{2}$, where the indices $p$ and $e$ denote physical values for protons and electrons, respectively, while $V_{e, p}$ denote the respective streaming velocities. Although this condition is realistic, since it implies less mobile protons $\left(V_{p} \simeq\left(m_{e} / m_{p}\right)^{1 / 2} V_{e} \ll V_{e}\right)$, we will relax this assumption in further studies. The new parametrization proposed here enables a better understanding of the interplay of counterstreaming and temperature anisotropy in the full range of plasma beta values. In Sec. V, we summarize the results, which can be relevant for the anisotropy constraints observed in the solar wind plasma. ${ }^{16,17}$

\section{DISPERSION RELATION}

We consider homogeneous nonrelativistic plasmas immersed into a uniform magnetic field, $\mathbf{B}=B_{0} \mathbf{e}_{z}$, with initially gyrotropic velocity distribution functions of the charged particles $f_{a, 0}(v)=f_{a, 0}\left(v_{\|}, v_{\perp}\right)$. The properties of the transverse electromagnetic wave modes propagating perpendicular to the magnetic field $\left(\mathbf{k}=k \mathbf{e}_{x}\right)$ are determined by the dispersion relation $^{18}$ 


$$
\operatorname{det}(\Lambda)=\operatorname{det}\left(\begin{array}{ccc}
1+R_{11} & R_{12} & R_{13} \\
-R_{12} & 1-\frac{k^{2} c^{2}}{\omega^{2}}+R_{22} & R_{23} \\
R_{13} & -R_{23} & 1-\frac{k^{2} c^{2}}{\omega^{2}}+R_{33}
\end{array}\right)=0
$$

where

$$
\begin{aligned}
& R_{11}=\sum_{a} 2 \pi \omega_{p, a}^{2} \frac{\Omega_{a}^{2}}{k^{2}} \int_{-\infty}^{\infty} d v_{\|} \int_{0}^{\infty} d v_{\perp} \sum_{n=-\infty}^{\infty} \frac{n^{2} J_{n}^{2}\left(z_{a}\right) \frac{\partial f_{a, 0}}{\partial v_{\perp}}}{\omega^{2}-n^{2} \Omega_{a}^{2}}, \\
& R_{12}=\sum_{a} 2 \pi i \omega_{p, a}^{2} \frac{\Omega_{a}^{3}}{\omega k^{2}} \int_{-\infty}^{\infty} d v_{\|} \int_{0}^{\infty} d v_{\perp} \\
& \times \sum_{n=-\infty}^{\infty} \frac{z_{a} n^{2} J_{n}\left(z_{a}\right) J^{\prime}{ }_{n}\left(z_{a}\right) \frac{\partial f_{a, 0}}{\partial v_{\perp}}}{\omega^{2}-n^{2} \Omega_{a}^{2}} \\
& R_{13}=\sum_{a} 2 \pi \omega_{p, a}^{2} \frac{\Omega_{a}^{2}}{\omega k} \int_{-\infty}^{\infty} d v_{\|} v_{\|} \int_{0}^{\infty} d v_{\perp} \sum_{n=-\infty}^{\infty} \frac{n^{2} J_{n}^{2}\left(z_{a}\right) \frac{\partial f_{a, 0}}{\partial v_{\perp}}}{\omega^{2}-n^{2} \Omega_{a}^{2}}, \\
& R_{22}=\sum_{a} 2 \pi \omega_{p, a}^{2} \frac{\Omega_{a}^{2}}{k^{2}} \int_{-\infty}^{\infty} d v_{\|} \int_{0}^{\infty} d v_{\perp} \sum_{n=-\infty}^{\infty} \frac{z_{a}^{2}\left(J^{\prime}{ }_{n}\left(z_{a}\right)\right)^{2} \frac{\partial f_{a, 0}}{\partial v_{\perp}}}{\omega^{2}-n^{2} \Omega_{a}^{2}}, \\
& R_{23}=-\sum_{a} 2 i \pi \omega_{p, a}^{2} \frac{\Omega_{a}}{k} \int_{-\infty}^{\infty} d v_{\|} v_{\|} \int_{0}^{\infty} d v_{\perp} \\
& \times \sum_{n=-\infty}^{\infty} \frac{z_{a} J_{n}\left(z_{a}\right) J^{\prime}{ }_{n}\left(z_{a}\right) \frac{\partial f_{a, 0}}{\partial v_{\perp}}}{\omega^{2}-n^{2} \Omega_{a}^{2}} \\
& R_{33}=-\sum_{a} \frac{\omega_{p, a}^{2}}{\omega^{2}}+\sum_{a} \frac{2 \pi \omega_{p, a}^{2}}{\omega^{2}} \int_{-\infty}^{\infty} d v_{\|} v_{\|}^{2} \int_{0}^{\infty} d v_{\perp} \\
& \times \sum_{n=-\infty}^{\infty} \frac{n^{2} \Omega_{a}^{2} J_{n}^{2}\left(z_{a}\right) \frac{\partial f_{a, 0}}{\partial v_{\perp}}}{\omega^{2}-n^{2} \Omega_{a}^{2}} .
\end{aligned}
$$

Here, $c$ is the speed of light in vacuum, $k$ is the wavenumber, $\omega_{p, a}=\sqrt{4 \pi q_{a}^{2} n_{a} / m_{a}}$ is the plasma frequency, and $\Omega_{a}=q_{a} B_{0} / m_{a} c$ is the (nonrelativistic) gyration frequency for different particle species ( $a=e$ for electrons, and $a=p$ for protons), and $J_{n}$ is the Bessel function of the first kind with the argument

$$
z_{a}=\frac{k v_{\perp}}{\Omega_{a}} .
$$

Now we introduce the initial distribution of particles

$$
f_{a, 0}=\sum_{s} \epsilon_{a, s} M\left(u_{a, \|, s}, u_{a, \perp}, V_{a, s}\right),
$$

consisting of counter-moving streams, each modeled by a drifting bi-Maxwellian distribution function

$$
\begin{aligned}
M\left(u_{a, \|, s}, u_{a, \perp}, \pm V_{a, s}\right)= & \frac{1}{(2 \pi)^{3 / 2} u_{a, \perp}^{2} u_{a, \|, s}} \exp \left(-\frac{v_{\perp}^{2}}{2 u_{a, \perp}^{2}}\right) \\
& \times \exp \left(-\frac{\left(v_{\|} \pm V_{a, s}\right)^{2}}{2 u_{a, \|, s}^{2}}\right) .
\end{aligned}
$$

Here, $V_{a, s}$ is the streaming velocity, $u_{a, \|, \perp, s}=\left(2 k_{B} T_{a, \|, \perp, s}\right)$ $\left.m_{a}\right)^{1 / 2}$ is the thermal velocity, and $\epsilon_{a, s} \equiv n_{a, s} / \sum_{s} n_{a, s}$ is the density of the stream $s$ normalized to the density of the entire plasma $\left(n_{a}=\sum_{s} n_{a, s}\right)$. Streams are assumed axially symmetric, with the same thermal motion in the transverse plane, i.e., $u_{a, \perp, s}=u_{a, \perp}$. Since

$$
\int_{-\infty}^{\infty} d v_{\|} \cdot v_{\|} M\left(u_{a, \|, s}, u_{a, \perp}, \pm V_{a, s}\right)=\frac{\mp V_{a, s}}{2 \pi u_{a, \perp}^{2}} \exp \left(-\frac{v_{\perp}^{2}}{2 u_{a, \perp}^{2}}\right),
$$

the nondiagonal elements $\Lambda_{13}, \Lambda_{23}, \Lambda_{31}$, and $\Lambda_{32}$ vanish for any species $a$ if their net current is neutral

$$
\sum_{s} \epsilon_{a, s} \mathbf{V}_{a, s}=0 .
$$

In contrast to previous investigations, which consider only neutral beams ${ }^{12} \quad\left(\epsilon_{e, s}=\epsilon_{p, s}, \mathbf{V}_{e, s}=\mathbf{V}_{p, s}\right)$, or immobile ions $^{11,19-21}\left(m_{i} \rightarrow \infty\right)$, the condition proposed in Eq. (12) is more general.

\section{A. The ordinary mode}

For a counterstreaming plasma satisfying condition (12), Eq. (1) provides the dispersion relation for the ordinary mode $\left(\mathbf{E} \| \mathbf{B}_{0}\right)$

$$
0=1-\frac{k^{2} c^{2}}{\omega^{2}}+R_{33} .
$$

Inserting $R_{33}$ from Eq. (7), the dispersion relation becomes

$$
\omega^{2}=b\left(\omega^{2}\right),
$$

where

$$
b\left(\omega^{2}\right)=c^{2} k^{2}+\sum_{a} \omega_{p, a}^{2}\left[1+\sum_{n=-\infty}^{\infty} \eta_{a}(n)\left(1+\frac{\omega^{2}}{n^{2} \Omega_{a}^{2}-\omega^{2}}\right)\right]
$$

and

$$
\eta_{a}(n)=2 \pi \int_{-\infty}^{\infty} d v_{\|} v_{\|}^{2} \int_{0}^{\infty} d v_{\perp} J_{n}^{2}(z) \frac{\partial f_{a, 0}}{\partial v_{\perp}} .
$$


Notice that $\eta_{a}(n)$ is not dependent on $\omega$, and negative if $Q\left(v_{\perp}\right)=\int_{-\infty}^{\infty} d v_{\|} v_{\|}^{2} f_{0}$ is monotonously decreasing. This is a characteristic feature of any separable distribution function

$$
f_{0}\left(v_{\perp}, v_{\|}\right)=f_{0, \perp}\left(v_{\perp}\right) f_{0, \|}\left(v_{\|}\right),
$$

with a monotonously decreasing transverse component $f_{0, \perp}\left(v_{\perp}\right)$. Our drifting-Maxwellian in Eq. (9) is an example of such a distribution function. Since $\eta_{a}(n)$ is negative, we use our earlier arguments ${ }^{23}$ that solutions of the dispersion relation (14) can only be either purely oscillating $(\Im(\omega)=0)$, or purely aperiodic $(\Re(\omega)=0)$.

By introducing the distribution function (9) and the modified Bessel function $I_{n}(x)=i^{-n} J_{n}(i x)$, which satisfies ${ }^{22}$

$$
\int_{0}^{\infty} \mathrm{d} z \cdot z e^{-\rho z^{2}} J_{n}^{2}(z)=\frac{e^{-\frac{1}{2 \rho}}}{2 \rho} I_{n}\left(\frac{1}{2 \rho}\right) .
$$

Equation (16) simplifies to

$$
\eta_{a}(n)=-\frac{U_{a}}{A_{a}} I_{n}\left(\frac{\xi_{a}^{2}}{2}\right) e^{-\frac{\xi_{a}^{2}}{2}}
$$

with the temperature anisotropy $A_{a}=u_{a, \perp}^{2} / u_{a, \|}^{2}$ and $\xi_{a}=\sqrt{2} k u_{a, \perp} / \Omega_{a}$. The quantity

$$
U_{a} \equiv C_{a}+\sum_{s} \epsilon_{a, s} \frac{V_{a, s}^{2}}{u_{a, \|}^{2}}=C_{a}+\frac{P_{a}}{\beta_{a \|}}
$$

represents the kinetic energy of the plasma streams where

$$
\begin{gathered}
\beta_{a \|} \equiv \frac{8 \pi n k_{B} T_{a \|}}{B_{0}^{2}} \quad C_{a} \equiv \sum_{s} \epsilon_{a, s} \chi_{a, s} \\
P_{a} \equiv \sum_{s} \epsilon_{a, s} \frac{2 \omega_{p, a}^{2}}{\Omega_{a}^{2}} \frac{V_{a, s}^{2}}{c^{2}} .
\end{gathered}
$$

As the parallel temperatures of the streams may be different, with $\chi_{a, s} \equiv u_{a, \|, s}^{2} / u_{a, \|}^{2}$, we normalize to an arbitrary quantity $u_{a, \|}^{2}$. This normalization can vary for different counterstreaming models (respectively for different applications), but has to be related to the thermal velocities of the streams. Obvious normalizations are, for example, $u_{a, \|}^{2}=\sum_{s} \epsilon_{a, s} u_{a, \|, s}^{2}$ (providing $C_{a}=1$ ) or the temperature of any stream $x$ (providing $u_{a, \|}^{2}=u_{a, \|, x}^{2}$ and $\left.\chi_{a, x}=1\right)$. For the special cases of symmetric streams with equal parallel temperatures $T_{a, \|, 1}=T_{a, \|, 2}$ $=T_{a, \|, 0} \equiv\left(m_{a} u_{a, \|, 0}^{2} / 2 k_{B}\right)^{1 / 2}$, we obtain the simplification $C_{a}=u_{a, \|, 0}^{2} / u_{a, \|}^{2}$. For a nonstreaming plasma $\left(V_{a, s}=0\right.$ respectively $P_{a}=0$ ), we simply find $C_{a}=1$ (as only the normalization to the only stream $u_{a, \|}=u_{a, \|, s}$ makes sense), and $U_{e}=1$ leading to the dispersion relation derived in earlier studies. ${ }^{23}$

For large frequencies, corresponding to $\omega^{2}>\Omega_{p}^{2}$, the dispersion relation (14) admits only stable solutions. In the opposite range $\omega^{2}<\Omega_{p}^{2}$, the new spectral parameter

$b(\omega=0) \equiv b_{0}(k)=k^{2} c^{2}+\sum_{a} \omega_{p, a}^{2}\left[1-\frac{1-I_{0}\left(\frac{\xi_{a}^{2}}{2}\right) e^{-\frac{\xi_{a}^{2}}{2}}}{A_{a}} U_{a}\right]$ provides the instability condition

$$
b_{0}(k) \leq 0
$$

and also an upper limit for the growth rate (i.e., $\Im(\omega) \equiv \omega_{i}$ )

$$
\omega_{i}^{2} \leq\left|b_{0}(k)\right|
$$

Although the mass ratio of the main plasma components (ions and electrons) is very large $m_{i} / m_{e} \geq m_{p} / m_{e} \simeq 1836 \gg 1$, in many cases of interest, parameters characterizing their free energies can be comparable, e.g., their temperatures $T_{e} \equiv m_{e} u_{e}^{2} /\left(2 k_{B}\right) \simeq T_{p} \equiv m_{p} u_{p}^{2} /\left(2 k_{B}\right) \quad$ (implying $\left.\quad \beta_{e} \simeq \beta_{p}\right)$, their anisotropies $A_{e} \simeq A_{p}$, and their streaming energies $U_{e} \simeq U_{p}$. These assumptions enable us to minimize the role of ions and build a simple analytical formalism. But according to the definition in Eq. (20), they also imply less mobile ions (protons), for instance, $V_{e} \simeq\left(m_{p} / m_{e}\right)^{1 / 2} V_{p} \gg V_{p}$.

\section{B. The instability condition}

The instability condition in Eq. (23) is a function of the wavenumber $k$. For the instability condition to be satisfied for an arbitrary $k$, we have to find the critical wave-number $k_{\text {min }}$ corresponding to the minimum

$$
b_{0, \min } \equiv b_{0}\left(k_{\min }\right) \leq b_{0}(k) \text {. }
$$

Only for negative values of $b_{0, \text { min }}<0$, growing modes can be found. To calculate $b_{0, \min }$, we take a closer look at the derivative of $b_{0}(k)$ (assuming equal parameters $\xi_{p} \simeq \xi_{e}$, $U_{p} \simeq U_{p}, \beta_{p} \simeq \beta_{e}$, and $\mu \equiv m_{p} / m_{e} \gg 1$ )

$$
\begin{aligned}
\frac{\partial b_{0}}{\partial k^{2}}=c^{2}\left[1+\frac{\left[I_{1}\left(\frac{\xi_{e}^{2}}{2}\right)-I_{0}\left(\frac{\xi_{e}^{2}}{2}\right)\right] e^{-\frac{\xi_{e}^{2}}{2}}}{2} \beta_{e \|} U_{e}\right. \\
\left.+\frac{\left[I_{1}\left(\frac{\xi_{e}^{2}}{2} \mu\right)-I_{0}\left(\frac{\xi_{e}^{2}}{2} \mu\right)\right] e^{-\frac{\xi_{e}^{2}}{2}} \mu}{2} \beta_{e \|} U_{e}\right] .
\end{aligned}
$$

For $\beta_{e \|} U_{e}<1$, the derivative is always positive and then the minimum $b_{0, \min }$ is found for $k_{\min }=0$. But according to $b_{0}(0)=\sum_{a} \omega_{p, a}^{2}>0$, the plasma is stable in this case. For all the other cases, we calculate $k_{\min }$ from the condition of minimum

$$
\frac{\partial b_{0}}{\partial k^{2}}=0
$$

and then the solution is inserted into Eq. (22) to find the instability threshold condition

$$
b_{0, \min }=0 \text {, }
$$

which sets the limit between stable and unstable configurations. Thus, the instability threshold conditions can be derived exactly by numerically solving the system of equations (27) and (28) (also derived before by Bornatici and Lee, ${ }^{11}$ but without proton term). Keeping the proton term, the instability conditions are 
provided in an accurate general form. Thresholds of the ordinary mode instability are displayed in Figs. 1 and 3 for different settings of the counterstreaming plasma system. These are calculated assuming $U_{e}$ dependent or independent of $\beta_{e \|}$.

\section{ASSUMING $U_{a}$ CONSTANT (NOT DEPENDENT ON $\beta_{a \|}$ )}

First, we consider counterstreaming configurations with the streaming parameter $U_{a}=$ constant, since this case has been evaluated numerically before, ${ }^{11,12}$ but, as far as we know, has not been described analytically. According to the definition (20), the analysis in this case explores the stability of a large variety of counterstream parameters consistent with $U_{a}=$ constant. These configurations can be found equally changing the streaming and thermal velocities, or varying the plasma beta parameter as only due to different particle densities or different magnetic field strengths.

\section{A. Stable plasma configurations}

As noted before, stable plasma configurations require $b_{0} \geq 0$, implying a sufficiently large anisotropy

$$
A_{a} \geq \frac{2 w_{a}^{2} U_{a}}{1+2 w_{a}^{2}}
$$

or sufficiently small values of the beta parameter

$$
\beta_{a \|} \leq \frac{1+2 w_{a}^{2}}{U_{a}}
$$

where $w_{a} \equiv \omega_{p, a} /(k c)$. For $U_{a}=1$, we retrieve the stability conditions for a nonstreaming plasma, ${ }^{23}$ but for larger values of $U_{a}>1$, the instability extends to smaller values of $\beta_{a, \|}<1$, and larger values of $A_{a}>1$ (i.e., when streams exhibit an excess of transverse temperature $T_{a, \perp}>T_{a, \|}$ ). For an anisotropic plasma with $A>1$, the ordinary mode would never grow unstable in the absence of streams $\left(U_{a}=1\right)$. This effect can be recognized in Fig. 1, where the unstable regimes are shown for different values of $U_{e}$.

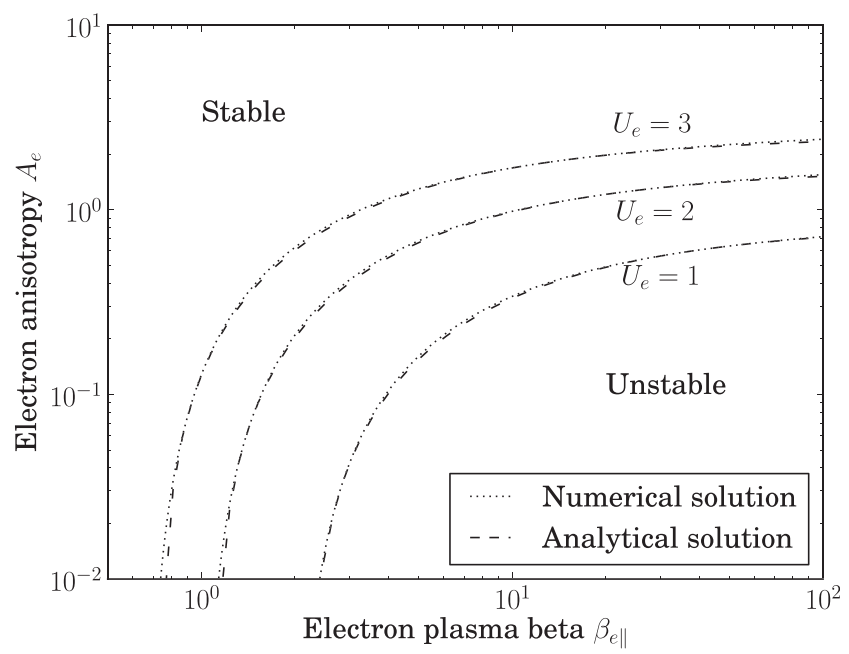

FIG. 1. Comparison of the exact instability thresholds derived numerically (dotted lines) and the new analytical approximation in Eq. (41) (dashed lines) for different values of $U_{e}$.

\section{B. The asymptotic limits of the instability condition}

Before deriving the analytical threshold condition in the whole range of parameters, we first examine the asymptotic limits $A_{e} \rightarrow 0$ and $\beta_{e \|} \rightarrow \infty$, as these cases can be derived without any approximations. In the limit of $A_{e} \rightarrow 0$, we find from Eq. (22)

$$
\left.b_{0}(k)\right|_{A_{e} \rightarrow 0}=\sum_{a} \omega_{p, a}^{2}+k^{2} c^{2}\left(1-\sum_{a} \frac{U_{a} \beta_{a \|}}{2}\right),
$$

and, assuming equal parameters of electrons and protons, the marginal condition $\left.b_{0}(k)\right|_{A_{e} \rightarrow 0}=0$ implies

$$
\beta_{e \|}=\frac{1}{U_{e}}+\sum_{a} \frac{\omega_{p, a}^{2}}{U_{e} k^{2} c^{2}} .
$$

But the threshold in Eq. (31) takes minimum (negative) values for a sufficiently large $U_{e} \beta_{e \|}>1$ and $k \rightarrow \infty$, implying

$$
\beta_{e \|}=\frac{1}{U_{e}}+\lim _{k \rightarrow \infty} \sum_{a} \frac{\omega_{p, a}^{2}}{U_{e} k^{2} c^{2}}=\frac{1}{U_{e}}
$$

for the marginal threshold condition $\left(b_{0, \min }=0\right)$ in the limit $A_{e} \rightarrow 0$.

In the limit of large plasma betas $\beta_{e \|} \rightarrow \infty$, we find (for equal parameters)

$$
\left.b_{0}(k)\right|_{\beta_{e \|} \rightarrow \infty}=k^{2} c^{2}+\left(1-\frac{U_{e}}{A_{e}}\right) \sum_{a} \omega_{p, a}^{2}
$$

and the marginal condition $b_{0}(k)=0$ reads

$$
A_{e}=U_{e} \frac{\sum_{a} \omega_{p, a}^{2}}{k^{2} c^{2}+\sum_{a} \omega_{p, a}^{2}} .
$$

In this limit, $b_{0}(k)$ takes minimum values for $k=0$, and then the marginal threshold condition $b_{0, \min }=0$ implies

$$
A_{e}=U_{e}
$$

For a nonstreaming plasma $\left(U_{e}=1\right)$, the asymptotic limits (33) and (36) become, respectively, $\beta_{e \|}=1$ and $A_{e}=1$. In the presence of streams $\left(U_{e}>1\right)$, the limits move to higher values of $A_{e}>1$ and smaller values of $\beta_{e \|}<1$ extending the instability regime. It should also be mentioned that the threshold conditions in the limit of $A_{e} \rightarrow 0$ in Eq. (33) do not match to those derived in Figs. 1 and 2 from Bornatici and Lee. ${ }^{11}$ Neglecting the proton term, Eq. (33) would read $\beta_{e \|}=2 / U_{e}$, (because of $U_{e} \beta_{e \|} / 2$ instead of $U_{e} \beta_{e \|}$ in Eq. (31)) leading to the values of Bornatici and Lee. ${ }^{11}$ But this difference is significant only for very large parallel temperatures $T_{\|} \gg T_{\perp}$ (i.e., $A_{e} \leq 10^{-2}$ ).

\section{Analytic description (neglecting proton contribution)}

Now, we derive analytical approximations for these threshold conditions, and compare them with the exact 
numerical evaluations in the range of interest. Here, we neglect the proton contribution in the system of Eqs. (27) and (28). To solve Eq. (27), we use the approximation $^{23}$

$$
\left[I_{1}(x)-I_{0}(x)\right] e^{-x} \simeq-u e^{-x},
$$

where the fitting parameter $u$ takes values in the interval $0.9 \leq u \leq 1$. With this approximation used in Eq. (26), the condition of minimum becomes

$$
\frac{\partial b_{0}}{\partial k^{2}} \simeq c^{2}\left[1-\frac{u}{2} e^{-\frac{\xi_{e}^{2}}{2}} \beta_{e \|} U_{e}\right]=0
$$

providing

$$
k_{\min }^{2}=\frac{2 \omega_{p, e}^{2}}{c^{2} \beta_{e \|} A_{e}} \ln \left(\frac{u}{2} \beta_{e \|} U_{e}\right) .
$$

Then from Eq. (22) we obtain

$$
\begin{aligned}
b_{0, \text { min }} \equiv b_{0}\left(k_{\min }\right)= & \omega_{p, e}^{2}\left(\frac{2 \ln \left(\frac{u}{2} \beta_{e \|} U_{e}\right)}{A_{e} \beta_{e \|}}+1\right. \\
& \left.-\frac{U_{e}-I_{0}\left(\ln \left(\frac{u}{2} \beta_{e \|} U_{e}\right)\right) \frac{2}{u \beta_{e \|}}}{A_{e}}\right) .
\end{aligned}
$$

Thus, the analytical expression for the marginal condition of stability $b_{0, \min }=0$ reads

$$
A_{e}=U_{e}-2 \frac{\ln \left(\frac{u}{2} \beta_{e \|} U_{e}\right)+\frac{1}{u} I_{0}\left(\ln \left(\frac{u}{2} \beta_{e \|} U_{e}\right)\right)}{\beta_{e \|}},
$$

which is displayed in Fig. 1. The best analytical approximation is found for a fitting parameter $u=0.9$ (dashed lines), which fits very well to the exact numerical conditions of marginal stability (dotted lines). Since the numerical condition is exactly calculated keeping the proton term, we conclude that the influence of protons is negligibly small, except in the case of nonphysically large anisotropies $\left(T_{\|} / T_{\perp}>10^{2}\right)$. The threshold conditions derived here for a counterstreaming plasma model have basically the same shape as those obtained for a nonstreaming plasma ${ }^{23}$ ( $U_{e}=1$ ), but increase with $U_{e}$ to higher values of the anisotropy $A_{e}$, and extend to smaller values of the plasma beta $\beta_{e \|}$. In both cases, the instability regime is significantly extended compared to the nonstreaming plasma.

Equations (40) and (41) only hold for $\beta_{e \|}>2 / U_{e}$. These results do not apply for smaller values of the plasma beta, as the derivative in Eq. (26) is always positive and $b_{0, \min }=b_{0}(0)>0$, only leading to stable plasma configurations.

\section{Isotropic counterstreams}

If the streams are isotropic, i.e., $T_{e \perp}=T_{e \|}$ and $A_{e}=1$, the instability threshold in Eq. (40) simplifies to

$$
\begin{aligned}
& b_{0, \min }=\omega_{p, e}^{2} \\
& \quad \times\left(2 \frac{u \ln \left(\frac{u}{2} \beta_{e \|} U_{e}\right)+I_{0}\left(\ln \left(\frac{u}{2} \beta_{e \|} U_{e}\right)\right)}{u \beta_{e \|}}+1-U_{e}\right)=0,
\end{aligned}
$$

which is illustrated in Fig. 2. The unstable plasma regimes are found above the plotted thresholds (the marginal condition of stability is displayed with a solid line). For large values of the plasma beta parameter, we obtain

$$
\lim _{\beta_{e \|} \rightarrow \infty} b_{0, \min }=\omega_{p, e}^{2}\left(1-U_{e}\right),
$$

providing

$$
U_{e, \text { asympt }}=1-\frac{b_{0, \min }}{\omega_{p, e}^{2}},
$$

with $U_{e, \text { asympt }}=1$ for marginal stability $b_{0, \min }=0$.

\section{ASSUMING $U_{a}$ DEPENDENT ON $\beta_{a} \|$}

In contrast to Sec. III, but in accord with the new parametrization in Eq. (20), now we assume that $U_{a}\left(P_{a}, \beta_{a, \|}\right)$ depends on $\beta_{a \|}$. The instability conditions are determined varying temperature values and their anisotropy, but keeping the streaming velocity and $n / B_{0}^{2}$ constant. Such an approach is relevant for the solar wind plasma diagnostics, in order to predict the temperature anisotropy at marginal stability.

\section{A. Stable plasma configurations}

For stable plasma configurations, the anisotropy condition takes the same form as in Eq. (29)

$$
A_{a} \geq \frac{2 w_{a}^{2} U_{a}}{1+2 w_{a}^{2}}=\frac{2 w_{a}^{2}}{1+2 w_{a}^{2}}\left(C_{a}+\frac{P_{a}}{\beta_{a \|}}\right),
$$

but now depends on $\beta_{a \|}$. Therefore, in the limit of $\beta_{a \|} \gg P_{a}$, we expect instability thresholds similar to those found for a

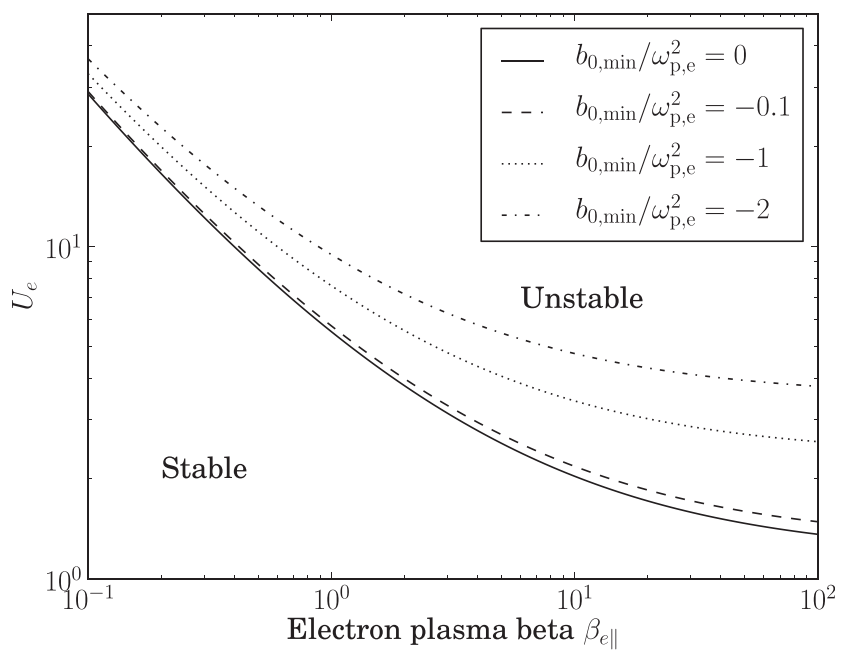

FIG. 2. The instability threshold for isotropic beams, as provided by Eq. (42) for different values of $b_{0, \min }$ (the maximum limits for the growth rate). 

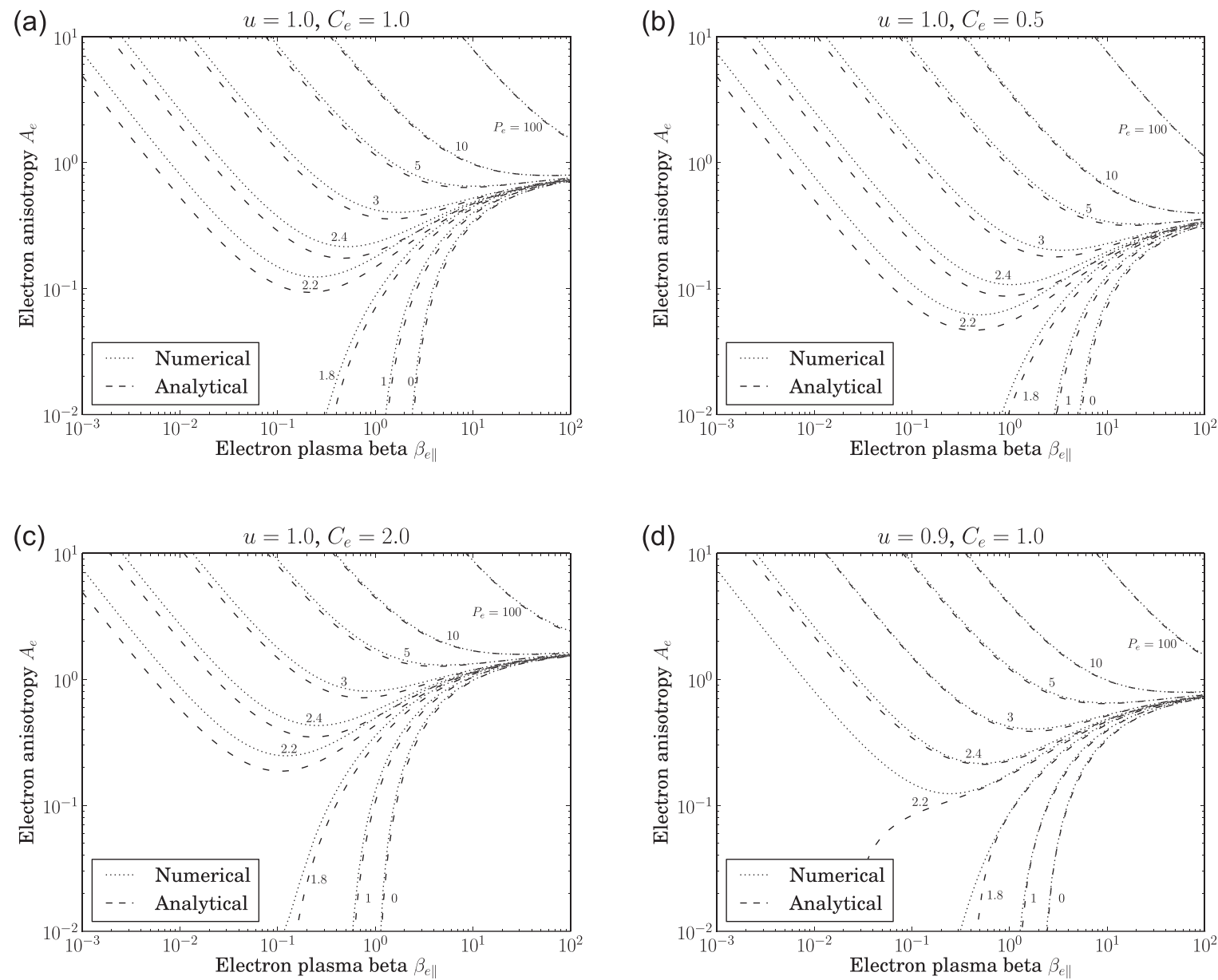

FIG. 3. Comparison of the instability thresholds derived exactly numerically (dotted lines) for different values of $P_{e}$ and $C_{e}$, and analytical approximations provided by Eq. (41) (dashed lines) for two values of the fitting parameter $u=0.9$ and $u=1.0$. The unstable states lie under the given threshold curve.

nonstreaming plasma ${ }^{23}$ (for $C_{a}=1$ ), but in the opposite plasma beta range $\beta_{a \|} \ll P_{a}$, the plasma is stabilized only if $T_{\perp} \gg T_{\|}$.

For stable configurations in terms of the plasma beta condition (30) changes to

$$
\beta_{a \|} \leq \frac{1-P_{a}+2 w_{a}^{2}}{C_{a}}
$$

For $P_{a}>1$, and sufficiently small wavenumbers, i.e., $2 w_{a}^{2}=2 \omega_{p, a}^{2} /(k c)^{2}<P_{a}-1$, counterstreaming plasmas can be unstable for any value of $\beta_{a \|}>0$. This is confirmed by the exact numerical evaluation of the instability thresholds (dotted lines) displayed in Fig. 3.

\section{B. The asymptotic limits of the instability condition}

For very large $\beta_{e \|} \rightarrow \infty$, the asymptotic limit of the instability threshold is provided in Eq. (36). But now, according to our assumptions, $U_{e}$ depends on $\beta_{e \|}$, and using the definition in Eq. (20) the limit of the instability threshold reduces to

$$
A_{e}=C_{e} .
$$

For very small anisotropies $A_{e} \rightarrow 0$, we find from Eq. (33)

$$
1=U_{e} \beta_{e \|}
$$

so that with Eq. (20), this limit provides

$$
\beta_{e \|}=\frac{1-P_{e}}{C_{e}} .
$$

We notice that $P_{e}=1$ is a critical value in this case, since the plasma beta cannot be negative.

We now derive more accurate estimations of the critical value $P_{e \text {,critical }}$, which determines if the threshold condition approaches $A_{e} \rightarrow 0$ or not, for finite values of $\beta_{e \|}>0$. For small values of $\beta_{e \|}<1$, the minimum of $b_{0}(k)$ is found for small $\xi_{e}^{2}<1$, implying that for $\beta_{e \|} \rightarrow 0$, we can write (approximately for Eq. (22))

$$
\begin{aligned}
b_{0}(k)= & k^{2} c^{2}+\sum_{a} \omega_{p, a}^{2}\left(1-\frac{\frac{\xi_{a}^{2}}{2}-\frac{3 \xi_{a}^{4}}{16}}{A_{a} \beta_{a \|}}\left(C_{a} \beta_{a \|}+P_{a}\right)\right) \\
= & k^{2} c^{2}+\sum_{a}\left(\omega_{p, a}^{2}-\frac{k^{2} c^{2}}{2}\left(C_{a} \beta_{a \|}+P_{a}\right)\right. \\
& \left.+\frac{3 \beta_{a \|} k^{4} c^{4}\left(C_{a} \beta_{a \|}+P_{a}\right)}{16 \omega_{p, a}^{2}} A_{a}\right) .
\end{aligned}
$$

Assuming equal parameters, the marginal condition $b_{0}(k)=0$ becomes 


$$
\begin{aligned}
A_{e}= & \frac{16 \omega_{p, p}^{2}}{3 \beta_{e \|} k^{4} c^{4}\left(C_{e} \beta_{e \|}+P_{e}\right)} \\
& \times\left[-k^{2} c^{2}-\sum_{a}\left(\omega_{p, a}^{2}-\frac{k^{2} c^{2}}{2}\left(C_{a} \beta_{a \|}+P_{a}\right)\right)\right] .
\end{aligned}
$$

As the threshold condition $b_{0, \min }$ is found for large values of $k^{2} \rightarrow \infty$, we obtain

$$
A_{e} \simeq \frac{16 \omega_{p, p}^{2}}{3 \beta_{e \|} k^{2} c^{2}\left(C_{e} \beta_{e \|}+P_{e}\right)}\left[-1+\sum_{a} \frac{C_{a} \beta_{a \|}+P_{a}}{2}\right]
$$

This explains the (nearly) linear dependence for small values of $\beta_{e \|}<1$ in the logarithmic plots of Fig. 3. For a given $k$, in the limit $\beta_{e \|} \rightarrow 0$, we find

$$
A_{e}=\lim _{\beta_{e} \rightarrow 0} \frac{\text { const. }}{\beta_{e}}\left[-1+\sum_{a} \frac{P_{a}}{2}\right] \rightarrow \infty \text { if } \sum_{a} P_{a}>2 .
$$

Thus, for equal parameters $P_{e}=P_{p}$, and sufficiently large values $P_{e}>P_{e \text {,critical }}=1$, the threshold becomes very large $A_{e} \rightarrow \infty\left(T_{\perp} \gg T_{\|}\right)$.

If the proton term is neglected we find again $A_{e} \rightarrow \infty$, but for $P_{e}>P_{e, \text { critical }}=2$. Remembering that we are in the limit of small $\beta_{e \|} \rightarrow 0$, the instability threshold in Eq. (53) is not defined below the critical value $P_{e, \text { critical }}$, because the anisotropy is defined positive $(A>0)$. For these small beta regimes, plots in Fig. 3 indicate no thresholds for $P_{e}<P_{e, \text { critical }}$.

\section{Analytic description}

Analytically, the instability threshold is again described by Eq. (41) with $U_{e}\left(\beta_{e \|}\right)$. In Fig. 3, the analytical approximations (dashed lines) are compared with the exact numerical thresholds (dotted lines). We find that $u=0.9$ provides again an accurate analytical approximation in Eq. (41), except for values close to $P_{e \text {,critical }} \simeq 2$, where, for small $\beta_{e \|}<1$ the analytical approximation does not fit with the exact thresholds. In the range of $P_{e} \simeq 2$, analytical thresholds can be calculated taking $u=1$, which provides a better fit with the numerical thresholds. Notice that the new analytical threshold in Eq. (41) has been found by minimizing the role of protons. This implies a departure from the exact threshold (see Sec. IV B), which is relevant only in the limit of very small $\beta_{e \|} \ll 10^{-3}$ and $A_{e} \ll 10^{-2}$.

The new parametrization proposed here enables us to perform a general approach of the ordinary mode instability, covering the regimes discussed in Sec. III, which are associated with small values of $P_{e}<P_{e, \text { critical, as well as the new }}$ regimes of instability associated with large values of $P_{e}>P_{e, \text { critical }}$. In both cases, the threshold condition is approaching $A_{e}=C_{e}$ (see Eq. (47)) for very large $\beta_{e \|} \rightarrow \infty$, and we obtain a minimum $A_{e, \min }\left(P_{e}\right)$ at $\beta_{e \|, \min }\left(P_{e}\right)$.

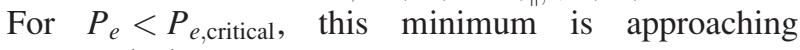
zero $\left(A_{e, \min }\left(P_{e}\right)=0\right)$ and we obtain unstable plasma configurations only in the range $\beta_{e \|}>\beta_{e \|, m i n}\left(P_{e}\right)$. Since $\beta_{e \|, \min }\left(P_{e}\right)=\left(1-P_{e}\right) / C_{e}$ (see Eq. (49)) the regime of instability extends for larger $P_{e}$.

In the opposite case $P_{e}>P_{e, \text { critical }}$, we obtain $A_{e, \text { min }}\left(P_{e}\right)>0$, implying that below this minimum (i.e., for $\left.A_{e}<A_{e, m i n}\right)$ the plasma is unstable for any value of the plasma beta. This minimum increases with $P_{e}$ and moves to larger values of $\beta_{e \|, m i n}\left(P_{e}\right)$, extending the regime of instability. For low plasma betas $\beta_{e \|}<\beta_{e \|, m i n}\left(P_{e}\right)$, the threshold increases exponentially to unlimited large values $A_{e} \gg 1$.

Hence, the ordinary mode is unstable for any value of the temperature anisotropy $A_{e}>0$, if the streaming parameter $P_{e}$ is large enough, and $\beta_{e, \|}$ is small enough. Less susceptible to the instability are only the upper limits of very large $T_{\perp} \gg T_{\|}$. Highly magnetized streams in the range of small $\beta_{e, \|}<1$, are not necessarily stable (see Fig. 3), as for large values of $P_{e}>P_{e, \text { critical }}$, their stability requires very large $T_{\perp} \gg T_{\|}$, and instabilities can be found for any value of $\beta_{e, \|}$. As expected, for moderately small values of the plasma beta $\beta_{e, \|} \simeq \beta_{e \|, \min }\left(P_{e}\right)$, the instability is enhanced by an excess of parallel temperature $T_{\|}>T_{\perp}$, but it can also be enhanced by a transverse compression $T_{\perp}>T_{\|}$(mirror-like) for even smaller values of $\beta_{e, \|} \ll 1$.

\section{Isotropic counterstreams}

For isotropic temperatures $\left(A_{e}=1\right)$ the threshold in Eq. (40) becomes

$$
b_{0, \min }=\omega_{p, e}^{2}\left(2 \frac{u \ln \left(\frac{u}{2} \beta_{e \|} C_{e}+\frac{u}{2} P_{e}\right)+I_{0}\left(\ln \left(\frac{u}{2} \beta_{e \|} C_{e}+\frac{u}{2} P_{e}\right)\right)-\frac{u}{2} P_{e}}{u \beta_{e \|}}+1-C_{e}\right) .
$$

These thresholds, including the marginal stability condition $b_{0, \text { min }}=0$ are displayed in Fig. 4. For sufficiently small values of $P_{e}<P_{e}\left(\beta_{e \|} \rightarrow 0\right)$, the counterstreams are stable for any value of $\beta_{e \|}$. Otherwise, for larger values of $P_{e}>P_{e}\left(\beta_{e \|} \rightarrow 0\right)$, (corresponding to higher streaming velocities $V_{a}$ ), the ordinary mode is unstable for sufficiently small $\beta_{e \|}$. This can also be recognized for anisotropic configurations in Fig. 3 at $A_{e}=1$.
In the limit of very small $\beta_{e \|} \rightarrow 0$, Eq. (54) becomes

$$
P_{e}=\frac{u \ln \left(\frac{u}{2} P_{e}\right)+I_{0}\left(\ln \left(\frac{u}{2} P_{e}\right)\right)}{\frac{u}{2}},
$$

which is independent of $b_{0, \min }$. As in this limit $u=1$ provides an exact description, we obtain 


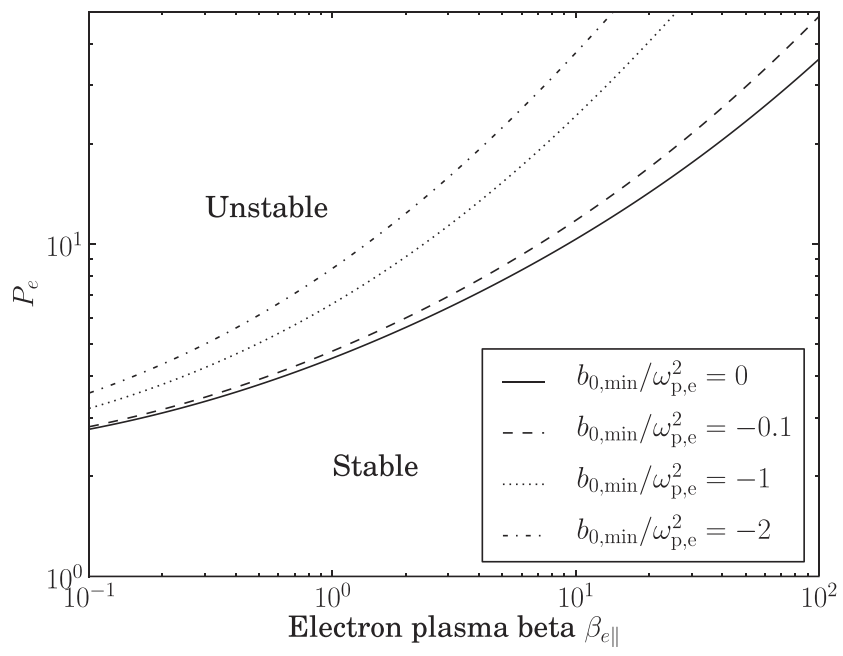

FIG. 4. The threshold conditions for isotropic beams, as provided by Eq. (54) for different values of $b_{0, \min }$.

$$
P_{e}=\frac{\ln \left(0.5 P_{e}\right)+I_{0}\left(\ln \left(0.5 P_{e}\right)\right)}{0.5}
$$

leading to $P_{e}\left(\beta_{e \|} \rightarrow 0\right)=2$. This quantity corresponds to $P_{e, \text { critical }}$ for anisotropic settings (see Fig. 3). The difference to the exact value $P_{e \text {,critical }}=1$ derived in Sec. IV B is a consequence of the minimized proton dynamics. Choosing $u=0.9$ leads to $P_{e, \text { critical }} \simeq 2.2$, which explains the misfits of the analytical and numerical thresholds in the interval $2 \leq P_{e} \leq 2.2$ in Fig. 3 .

\section{CONCLUSIONS AND DISCUSSIONS}

The ordinary mode can be driven unstable by drifting bi-Maxwellian plasma particle distributions with and without temperature anisotropy. For a nonstreaming plasma, accurate analytical forms of the instability conditions have recently been derived for the full physical range of the temperature anisotropy and the parallel plasma beta. ${ }^{23}$ For countermoving plasma streams, the instability remains purely growing $(\Re(\omega)=0)$, but markedly extends to lower plasma beta regimes. This is relevant for the explanation of the observed limits of stable plasma configurations in the solar wind $^{16,17,24}$ and the magnetosphere. ${ }^{25}$

In order to refine the analysis of the ordinary mode, we adopt realistic settings, assuming counterstreaming plasmas are magnetized and hot enough, and their temperature is not necessarily isotropic. In Sec. II, we propose a new parametrization, which enables us to understand the interplay of counterstreaming and temperature anisotropy and to derive accurate analytical forms of the instability thresholds for all plasma beta parameter. Thus, the instability condition, usually constraining only the temperature anisotropy and the plasma beta, now becomes explicitly dependent on the streaming velocity and the parallel thermal velocity through the new parameters $U$ and $P$ defined in Eqs. (20) and (21). We have investigated two complementary cases: first, considering counterstreaming configurations with the parameter $U=$ constant, not dependent on $\beta_{\|}$ (in Sec. III). Secondly, we have taken $U$ dependent on $\beta_{\|}$ (in Sec. IV).

In Sec. III, we provide an accurate analytical description of the ordinary mode instability for a counterstreaming plasma. For counterstreams with bi-Maxwellian distributions, the instability condition is described by Eq. (41) for any value of the temperature anisotropy and the (nonrelativistic) streaming velocity. In Sec. IV, we have found new regimes of the ordinary mode instability, investigating the full range of (nonrelativistic) values for the temperature anisotropy, the streaming velocity and the plasma beta parameter. Both, the exact numerical threshold conditions and their analytical approximations, indicate the presence of this instability for all values of the plasma beta parameter $\left(\beta_{\|}>0\right)$. The very low beta regime $\left(\beta_{\|}<1\right)$ is unstable, if the streaming velocity is large enough. These results may therefore be relevant for the solar wind plasma diagnostics, in order to predict the temperature anisotropy and/or the energy of streams close to the marginal stability condition in the low plasma-beta regimes.

We demonstrated that the new analytical approximation (41) provides an accurate description for the instability threshold in the whole range of $\beta_{\|}$. Limiting cases, including isotropic temperatures $\left(T_{\|} \simeq T_{\perp}\right)$, very large anisotropy $\left(T_{\|} \gg T_{\perp}\right)$, and very large plasma beta values $\left(\beta_{\|} \rightarrow \infty\right)$, have been analyzed in detail. To minimize the role of protons, we have adopted equal parameters of electrons and protons, characterizing their free energy, e.g., $T_{e} \simeq T_{p}$ and $U_{e} \simeq U_{p}$, which imply $V_{e} \simeq\left(m_{p} / m_{e}\right)^{1 / 2} V_{p} . \gg V_{p}$. This restriction will be relaxed in the second paper, adding the effects of ions.

\section{ACKNOWLEDGMENTS}

We thank the referee for constructive and helpful criticism. The authors acknowledge partial support from the Deutsche Forschungsgemeinschaft through Grant Nos. Schl 201/21-2 and Schl 201/25-1. M.L. also acknowledges financial support from the EU Commission and Research Foundation Flanders (FWO) as FWO Pegasus Marie Curie Fellow (Grant No. 1.2.070.13).

${ }^{1}$ R. Schlickeiser and P. K. Shukla, Astrophys. J. 599, L57 (2003).

${ }^{2}$ A. Bret, M.-C. Firpo, and C. Deutsch, Phys. Rev. Lett. 94, 115002 (2005).

${ }^{3}$ A. Bret, L. Gremillet, D. Benisti, and E. Lefebvre, Phys. Rev. Lett. 100, 205008 (2008).

${ }^{4}$ A. Bret, Astrophys. J. 699, 990 (2009).

${ }^{5}$ A. Bret, L. Gremillet, and M. E. Dieckmann, Phys. Plasmas 17, 120501 (2010).

${ }^{6}$ M. Lazar, R. Schlickeiser, R. Wielebinski, and S. Poedts, Astrophys. J. 693, 1133 (2009)

${ }^{7}$ A. Bret and C. Deutsch, Phys. Plasmas 13, 022110 (2006).

${ }^{8}$ R. Schlickeiser, M. Lazar, and T. Skoda, Phys. Plasmas 18, 012103 (2011).

${ }^{9}$ R. Schlickeiser and T. Skoda, Astrophys. J. 716, 1596 (2010).

${ }^{10}$ A. Stockem, I. Lerche, and R. Schlickeiser, Astrophys. J. 651, 584 (2006).

${ }^{11}$ M. Bornatici and K. F. Lee, Phys. Fluids 13, 3007 (1970).

${ }^{12}$ K. F. Lee and J. C. Armstrong, Phys. Rev. A 4, 2087 (1971).

${ }^{13}$ R. Tautz and R. Schlickeiser, Phys. Plasmas 13, 062901 (2006).

${ }^{14}$ M. Lazar, R. C. Tautz, R. Schlickeiser, and S. Poedts, Mon. Not. R. Astron. Soc. 401, 362 (2010).

${ }^{15}$ R. C. Davidson, in Handbook of Plasma Physics, edited by M. N. Rosenbluth and R. Z. Sagdeev (North-Holland, Amsterdam, 1983), Vol. 1, p. 519. 
${ }^{16}$ S. Stverak, P. Travnicek, M. Maksimovic, E. Marsch, A. N. Fazakerley, and E. E. Scime, J. Geophys. Res. 113, A03103, doi:10.1029/ 2007JA012733 (2008).

${ }^{17}$ S. D. Bale, J. C. Kasper, G. G. Howes, E. Quataert, C. Salem, and D. Sundkvist, Phys. Rev. Lett. 103, 211101 (2009).

${ }^{18}$ R. Schlickeiser, Cosmic Ray Astrophysics (Springer, Berlin, 2002).

${ }^{19}$ K. F. Lee, Phys. Rev. 181, 447 (1969).

${ }^{20}$ K. F. Lee, Phys. Rev. 181, 453 (1969).
${ }^{21}$ K. F. Lee, Phys. Rev. Lett. 21, 1439 (1968).

${ }^{22}$ I. S. Gradshteyn and I. N. Ryzhik, Table of Integrals, Series and Products (Academic, 2007).

${ }^{23}$ D. Ibscher, M. Lazar, and R. Schlickeiser, Phys. Plasmas 19, 072116 (2012).

${ }^{24}$ B. A. Maruca, J. C. Kasper, and S. D. Bale, Phys. Rev. Lett. 107, 201101 (2011).

${ }^{25}$ Z. Vörös, Nonlinear Processes Geophys. 18, 861 (2011). 